\section{The UbcH10 gene is a novel therapeutic target in anaplastic thyroid carcinoma}

The discovery of a 150 -fold upregulation of the $\mathrm{UbcH} 10$ gene in human thyroid carcinoma cell lines of varying histotype was the focus of a recent analysis by Pallante et al., published in the British Journal of Cancer. The group investigated the role of $\mathrm{UbcH} 10$ in thyroid carcinogenesis and proposed that the $\mathrm{UbcH} 10$ protein might represent a novel tumor marker and a therapeutic target in anaplastic thyroid carcinoma.

Immunohistochemistry, western blot analysis and reverse transcription polymerase chain reaction were used to determine whether UbcH10 overexpression was found in thyroid tumors as well as in cultured thyroid carcinoma cell lines. The highest levels of $\mathrm{UbcH} 10$ were found in anaplastic carcinomas (median positive cells $45.8 \%$ ) with noticeable levels in poorly differentiated, follicular and papillary carcinomas, and weak expression in follicular adenomas. Normal thyroid, nodular goiter and Hashimoto's thyroiditis were negative for $\mathrm{UbcH} 10$. These results were confirmed by analysis of mouse tumor experimental models. The researchers also found a significant correlation $(P<0.001)$ between $\mathrm{UbcH} 10$ and tissue proliferation, using the proliferation marker Ki-67. Furthermore, suppression of $\mathrm{UbcH} 10$ protein synthesis in two thyroid carcinoma cell lines resulted in significant inhibition of cell growth.

The authors concluded that abundant $\mathrm{UbcH} 10$ is a feature of the aggressive anaplastic thyroid carcinoma histotype, and that suppression of $\mathrm{HbcH} 10$ synthesis or function could form the basis of future therapies.

Alexandra King

Original article Pallante P et al. (2005) UbcH10 overexpression may represent a marker of anaplastic thyroid carcinomas. Br J Cancer 93: 464-471

\section{Sensitivity to preoperative chemotherapy varies between breast cancer subtypes}

Response to treatment can vary considerably among histologically similar breast cancers. Cancer subtypes have been identified and broadly classified into four categories on the basis of their gene expression profiles. Rouzier et al. examined fine-needle aspiration samples from 82 women with breast cancer, who subsequently underwent 24 weeks of preoperative paclitaxel and doxorubicin-containing chemotherapy. A previously identified 'breast intrinsic' set of 534 genes was used for expression profiling. Tumors were classified into four molecular classes: luminal $(n=30)$, normal-like $(n=10)$ basal-like $(n=22)$ and erbB2+ $(n=20)$ subtypes. These subgroups had different sensitivities to preoperative chemotherapy, with basal-like and erbB2+ subgroups showing the highest rates of pathological complete response (pCR; both $45 \%$; 95\% Cl 23-68\%) and luminal and normallike subgroups showing the lowest rates (luminal $6 \%, 95 \% \mathrm{Cl} 1-21 \%$; normal-like, no pCR, 95\% Cl 0-31\%).

The pCR could be predicted from this subtype classification with equivalent accuracy to that obtained with conventional clinicopathological parameters. Logistic regression model analysis revealed that the molecular subgroups did not improve prediction accuracy compared with conventional parameters. The authors comment that this was because basal-like tumors were almost all estrogenreceptor negative, and erbB2+ and basal-like tumors were predominantly high gradecharacteristics associated with a higher $\mathrm{pCR}$.

Importantly, the genes that were associated with $\mathrm{pCR}$ in the basal-like and erbB2+ groups did not overlap. This suggests that the mechanisms of sensitivity to chemotherapy vary between these tumor subtypes.

Caroline Barranco

Original article Rouzier R et al. (2005) Breast cancer molecular subtypes respond differently to preoperative chemotherapy. Clin Cancer Res 11: 5678-5685

\section{A novel preoperative staging technique for NSCLC could prevent unnecessary surgery}

A high proportion of patients with non-smallcell lung cancer (NSCLC) undergo unnecessary thoracotomies because of the limited accuracy of current preoperative staging techniques. A recent prospective, nonrandomized trial conducted by a research group from The Netherlands aimed to address this issue by comparing the novel technique of transesophageal ultrasound-guided fine-needle aspiration (EUS-FNA) with mediastinoscopy for preoperative staging of NSCLC. The analysis, 